\title{
EL CONTROL INKA DE LAS FRONTERAS ÉTNICAS: REFLEXIONES DESDE EL VALLE DE RICRÁN EN LA SIERRA CENTRAL DEL PERÚ
}

\author{
THE INKA CONTROL OF ETHNIC FRONTIERS: THOUGHTS FROM \\ THE RICRÁN VALLEY IN THE CENTRAL HIGHLANDS OF PERU
}

\author{
Manuel F. Perales Munguía*
}

\begin{abstract}
Se presenta un sitio de probable filiación Inka identificado como Guayoay Vilca, huaca principal de la etnia Tarama (Albornoz 1967:30) en la montaña de Apohuayhuay, sierra central del Perú. Se revisa el panorama étnico durante el período Intermedio Tardío confrontando evidencias etnohistóricas y arqueológicas, con miras a identificar una posible zona de frontera entre las etnias Tarama y Xauxa. Se muestra que la ubicación del macizo de Apohuayhuay está en clara asociación con dos zonas de frontera, una cultural entre estas dos entidades étnicas, y otra natural que separa la sierra de Ricrán y la selva de Monobamba, ambas de profunda connotación simbólica para las poblaciones locales. Finalmente, se plantea la posible manipulación de esta doble frontera por parte de los Inka con fines de dominio ideológico mediante la edificación del sitio de Otorongo.
\end{abstract}

Palabras claves: sitio ceremonial Inka, Andes Centrales, valle de Ricrán, montaña de Apohuayhuay, frontera étnica.

This article deals with a possible Inka site known as Guayoay Vilca located near the main huaca of the Tarama ethnic group (Albornoz 1967:30) on Mt. Apohuayhuay in the central highlands of Peru. First I provide an overview of the ethnic groups found in this area during the Late Intermediate period utilizing both ethnohistorical and archaeological data. The evidence suggests that an ethnic boundary existed in this area between the Tarama and Xauxa. The snow-capped Mt. Apohuayhuy is clearly associated with two kinds of frontiers. The first is cultural, marking the division between the Xauxa and Tarama ethnic groups; the second is natural insofar as it stands at the nexus of two distinct landscapes: the highlands of Ricrán and the tropical forests of Monobamba. Both types of frontiers would have been of considerable symbolic significance to the local populations. I then discuss how the Inka may have attempted to manipulate this border in order to consolidate their ideological and political control over the native societies through their construction of the site of Otorongo.

Key words: Inka ceremonial site, Central Andes, Ricrán valley, Apohuayhuay, settlement patterns, ethnic frontiers.

Las investigaciones arqueológicas sobre la expansión del imperio Inka vienen otorgando mayor interés al rol de la implantación de una ideología de poder transmitida mediante actividades ceremoniales y manipulación de símbolos visuales de gran arraigo en los Andes, como aquellos relacionados a la fijación de fronteras (Fioravanti 1986-87). Al respecto Morris (1998) ha enfatizado en la importancia de la arquitectura pública Inka y la organización espacial de los sitios estatales como símbolos del régimen oficial y como mecanismos para la creación de un nuevo orden social, político y económico entre las poblaciones conquistadas que consideró el manejo de los conflictos interétnicos.

Uno de los símbolos oficiales de mayor prestigio en el Tahuantinsuyu fue el felino, el cual, en su modalidad de otorongo o jaguar, estuvo vinculado a las huacas del Antisuyu (Guamán Poma 1980 [1615]) que a su vez marcaban la frontera entre la sierra y la selva con una profunda connotación ideológica, tal como sucede con la montaña de Apohuayhuay $(5.106 \mathrm{msm})$ en la cuenca alta del río Ricrán, Perú central. Este rasgo geográfico, además, estaría comprometiendo una zona de frontera durante el Intermedio Tardío entre dos entidades étnicas mayores, Xauxa y Tarama, conocidas gracias a la documentación etnohistórica temprana. La asociación a esta montaña de un sitio de posible filiación Inka plantea la posibilidad de una manipulación ideológica de esta frontera por parte del régimen del Cuzco para consolidar su dominio en la región.

La cuenca del río Ricrán se localiza entre los $11^{\circ} 20^{\prime}$ y $11^{\circ} 39^{\prime}$ de latitud sur y $\operatorname{los} 75^{\circ} 25^{\prime}$ y $75^{\circ}$ $40^{\prime}$ de longitud occidental y su parte superior se

\footnotetext{
* Universidad Nacional Mayor de San Marcos, Lima. Museo Arqueológico “Julio Espejo Núñez” de Jauja. Jirón Panamá 1842, El Tambo-Huancayo, Perú. huacrash@yahoo.com, manuel.perales@gmail.com
} 
halla delimitada por el este por una sección de la cadena central de los Andes de cuyos deshielos se alimentan un sinnúmero de lagunas y cursos de agua que descienden hacia el valle de Ricrán y la selva de Monobamba. En este territorio se pueden identificar tres pisos ecológicos principales: janca o cordillera, puna y suni/quechua (Pulgar 1967) ${ }^{1}$. La puna comprende a los pastizales que se elevan por encima de los $4.000 \mathrm{msm}$ y la zona suni/quechua el fondo del valle $(3.600 \mathrm{msm}$.) y los sectores inferiores de las laderas que lo encierran. Nuestros trabajos se han concentrado en el territorio comprendido entre las actuales poblaciones de Chúlec- Janchiscocha y Congas-Antacucho, incluyendo el flanco occidental de la cordillera de Apohuayhuay en donde desde 1999 venimos desarrollando reconocimientos sistemáticos orientados al estudio de los patrones de asentamiento prehispánicos (Figura 1).

\section{La Configuración Étnica Regional Durante el Período Intermedio Tardío}

De acuerdo a la documentación del siglo XVI, la sierra central peruana comprendida dentro del actual departamento de Junín estuvo habitada por las etnias Chinchaycocha, Tarama, Xauxa, Huanca y Chuncu. No tenemos muchos detalles acerca de sus formas de organización sociopolítica y económica durante el Intermedio Tardío, aunque los testimonios recogidos por Andrés de Vega (1881 [1582]) sugieren un clima de fragmentación política, lo cual ha sido corroborado por las investigaciones arqueológicas (Bonnier 1997; D’Altroy 1981, 1992; Earle et al. 1987; Parsons et al. 2000).

Los Chinchaycocha ocuparon el altiplano de Junín y estuvieron ligados a una actividad ganadera que contrastó con la agricultura que se desarrolló en la región de Tarma, ocupada por el grupo

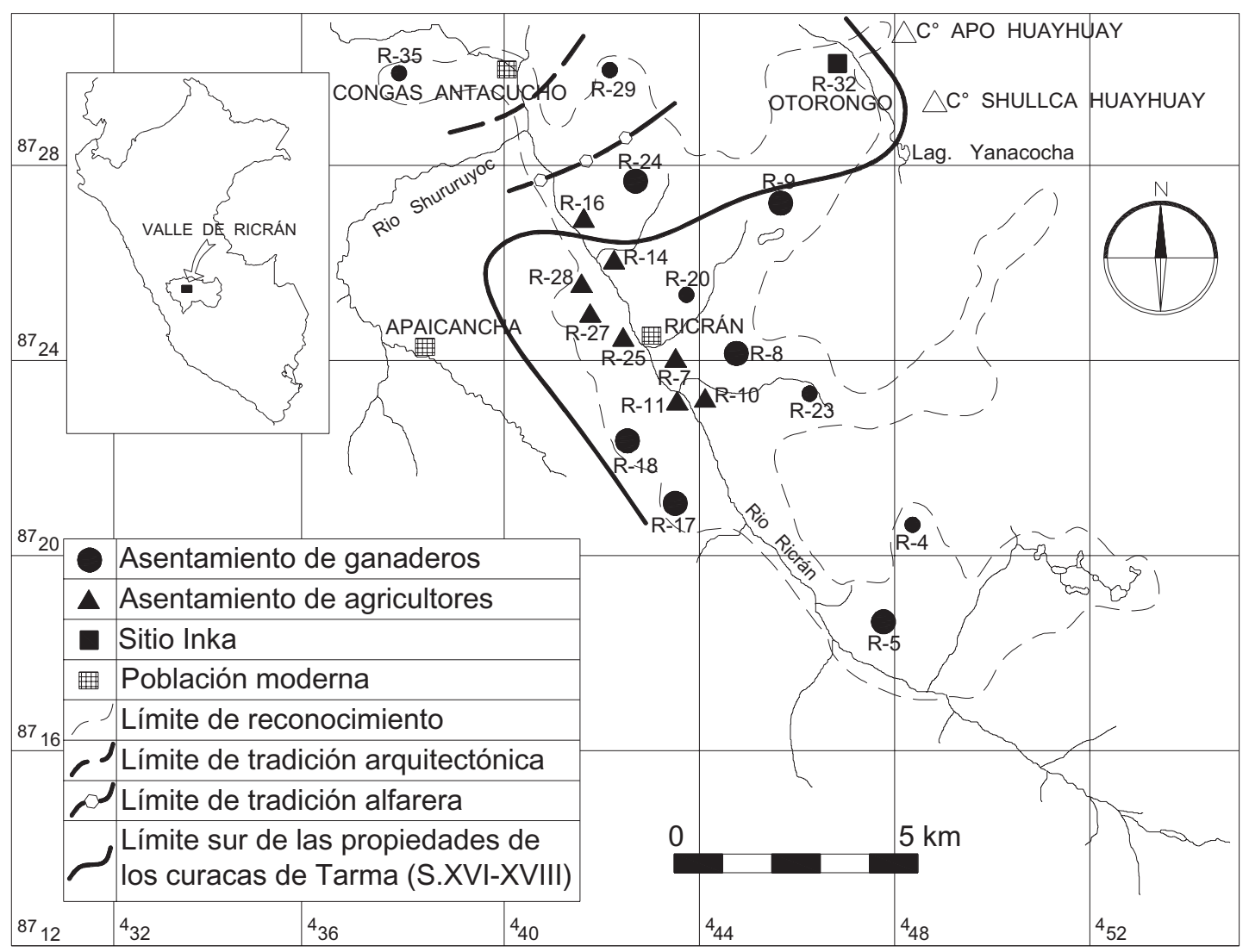

Figura 1. Asentamientos del Intermedio Tardío/Horizonte Tardío en el alto Ricrán y localización de la posible frontera XauxaTarama y del sitio de Otorongo (basado en la Carta Nacional IGN 1:100 000, hojas 23-1, 23-m, 24-1, 24-m, y Arellano 1988). Late Intermediate Period/Late Horizon settlements in the upper Ricrán valley, the location of the possible Xauxa-Tarama frontier, and the site of Otorongo (based on the Carta Nacional IGN 1:100 000, sheets 23-l, 23-m, 24-l, 24-m, and Arellano 1988). 
étnico Tarama (Cieza de León 1946 [1553], Cap. LXXXIII; Estete 1946 [1533]:101). Referencias etnohistóricas señalan vínculos entre ambas unidades étnicas bajo la administración Inka, por lo cual en la Colonia fueron consideradas dentro de una sola unidad administrativa (Arellano 1988; Hastings 1987; Matos et al. 1996). La naturaleza de estos vínculos es problemática ${ }^{2}$, sin embargo, arqueológicamente se muestran algunas relaciones entre los Chinchaycocha y Tarama durante el Intermedio Tardío como el uso de la cerámica San Blas Rojo sobre Ante (Hastings 1987; Parsons et al. 2000). Por su parte, los Xauxa y Huanca habitaron el valle del Mantaro, entre las actuales ciudades de Jauja y Huancayo ${ }^{3}$. Según la arqueología, habrían constituido dos grupos con numerosa población y con un nivel superior de organización sociopolítica que sus vecinos del norte y del sur (D’Altroy 1992; Parsons y Hastings 1988), tal como se puede apreciar en la mayor complejidad de sus asentamientos (Bonnier 1997) ${ }^{4}$. En cuanto a los Chuncu, éstos son mencionados por los curacas del valle del Mantaro en el siglo XVI (Vega 1881 [1582]), dando a entender su presencia durante tiempos anteriores al arribo de los Inkas a la región. Según Favre (1983) se habrían asentado hacia la cuenca del río Canipaco en los límites entre los departamentos de Junín y Huancavelica y de acuerdo a la escasa información arqueológica disponible habrían compartido muchos rasgos de su cultura material con los Huanca (Perales y Rodríguez 2003) $)^{5}$.

\section{La Frontera Xauxa-Tarama en Ricrán}

\section{Perspectiva etnohistórica}

Todavía es escasa la documentación publicada en relación al ámbito territorial de los Xauxa y Tarama. La demarcación eclesiástica de la sierra central durante el siglo XVIII incluye a Ricrán como parte del curato de Jauja (Espinoza 1973). Ello, sumado a la existencia temprana de la reducción de indígenas de Monobamba al este de Ricrán en la segunda mitad del siglo XVI y dentro de la parcialidad de Hatun Xauxa (Vega 1881 [1582]), indicaría un vínculo étnico prehispánico entre las poblaciones del alto Ricrán y Jauja. Por su parte, Carmen Arellano (1988) ha sugerido la existencia de la pichcapachaca de Kunkas en la parcialidad de Anantarma, cuyo territorio se habría localizado en torno al actual pueblo de Congas-Antacucho, a $6 \mathrm{~km}$ al NW del pueblo de Ricrán y también señala que las antiguas haciendas de Apaicancha, Ayas, Maco, Apaicanchilla y Yuracmayo estuvieron bajo la jurisdicción de los curacas de Tarma hasta los siglos XVII y XVIII ${ }^{6}$. Los linderos de esta última alcanzaban el pico de Apohuayhuay (Figura 1) que podría identificarse con "Guayoay vilca, guaca prencipal de los taramas... [y que] ...es un cerro questá frontero del tambo de Tarama" (Albornoz 1967:30), tal como lo ha propuesto también Hastings (1985:194). En síntesis la información etnohistórica sugiere la existencia en tiempos tardíos de una posible zona de frontera entre grupos vinculados a los Xauxa y Tarama localizada entre los actuales pueblos de Congas Antacucho y Ricrán (Figura 1).

\section{Perspectiva arqueológica}

Patrones de asentamiento y arquitectura. Durante el Intermedio Tardío casi todos los asentamientos en el alto Ricrán se localizaron hacia partes elevadas como crestas montañosas y estaban constituidos por concentraciones de estructuras circulares de piedra y barro distribuidas sobre terrazas artificiales, no observándose espacios centrales a modo de plazas como sí ocurre en el valle de Yanamarca, Jauja (Bonnier 1997; Earle et al. $1987)^{7}$. Tentativamente estos sitios pueden agruparse en dos categorías de acuerdo a su emplazamiento ecológico y rasgos asociados. A aquellos localizados sobre cumbres elevadas a más de 4.200 msm y que presentan muros perimétricos, zanjas y grandes corrales de piedra, con extensiones entre 14.0 y 1.5 há y fácil acceso a pastizales, los estamos catalogando como "asentamientos de ganaderos". Por otro lado, los sitios que se hallan en zonas más bajas, sobre estribaciones cordilleranas que descienden hacia el fondo del valle, entre los 3.800 y $4.000 \mathrm{msm}$, con extensiones entre 4.5 y 1.2 há y clara asociación a terrazas de cultivo, están siendo clasificados como "asentamientos de agricultores". Ahora bien, hay que destacar que en los sitios de la parte norte de nuestra área de estudio se observan estructuras de planta rectangular de dos niveles que reúnen las características típicas de los edificios que Parsons et al. $(1997,2000)$ consideran diagnósticos de la tradición arquitectónica del alto Tarma vinculada al grupo étnico Tarama (Figura 1$)^{8}$, la misma que se distingue de la 
arquitectura vinculada con los Xauxa que emplea de forma casi exclusiva la planta circular de un solo nivel (DeMarrais 2001).

Cerámica. Las secuencias alfareras elaboradas para la sierra central del Perú desde la década de 1960 (e.g., Browman 1970; Costin 1986; D' Altroy 1992; Earle et al. 1987; Hastorf et al. 1989; Lavallée 1967; LeBlanc 1981), no obstante los problemas que encierran, constituyen herramientas útiles para ubicar provisionalmente a los tipos alfareros del alto Ricrán dentro del contexto rsegional. En este sentido podemos adelantar que el componente cerámico más característico se vincula con el tipo Mantaro Base Clara, bastante popular en Jauja (Costin 1986, 1986-87; Lumbreras 1960; Matos 1959) $)^{9}$. Sin embargo, en algunos sitios localizados hacia los límites septentrionales de nuestra área de reconocimiento se ha registrado cerámica San Blas Rojo sobre Ante oriunda de la re- gión de Tarma y Chinchaycocha (Hastings 1985, 1987; Parsons et al. 1997, 2000) $)^{10}$.

En general, estas evidencias sugieren una zona de cambios en la cultura material de las poblaciones locales tardías del alto Ricrán, principalmente en su arquitectura y cerámica, hacia el territorio donde hoy se localizan los pueblos de Congas Antacucho y Maco, a unos 6 km al NW del pueblo de Ricrán (Figura 1).

\section{Evidencias Asociadas a la Montaña de Apohuayhuay: el Sitio de Otorongo (R-32)}

Se localiza en la margen izquierda del río Opamayo, a $4.060 \mathrm{msm}$, en el límite entre los territorios de las actuales comunidades campesinas de Ricrán y Yuracmayo. Sus coordenadas UTM son 446800 E y $8729780 \mathrm{~N}$ y ocupa el fondo de una quebrada amplia, en la base de un farallón de roca calcárea (Figura 2). El sitio consiste en un

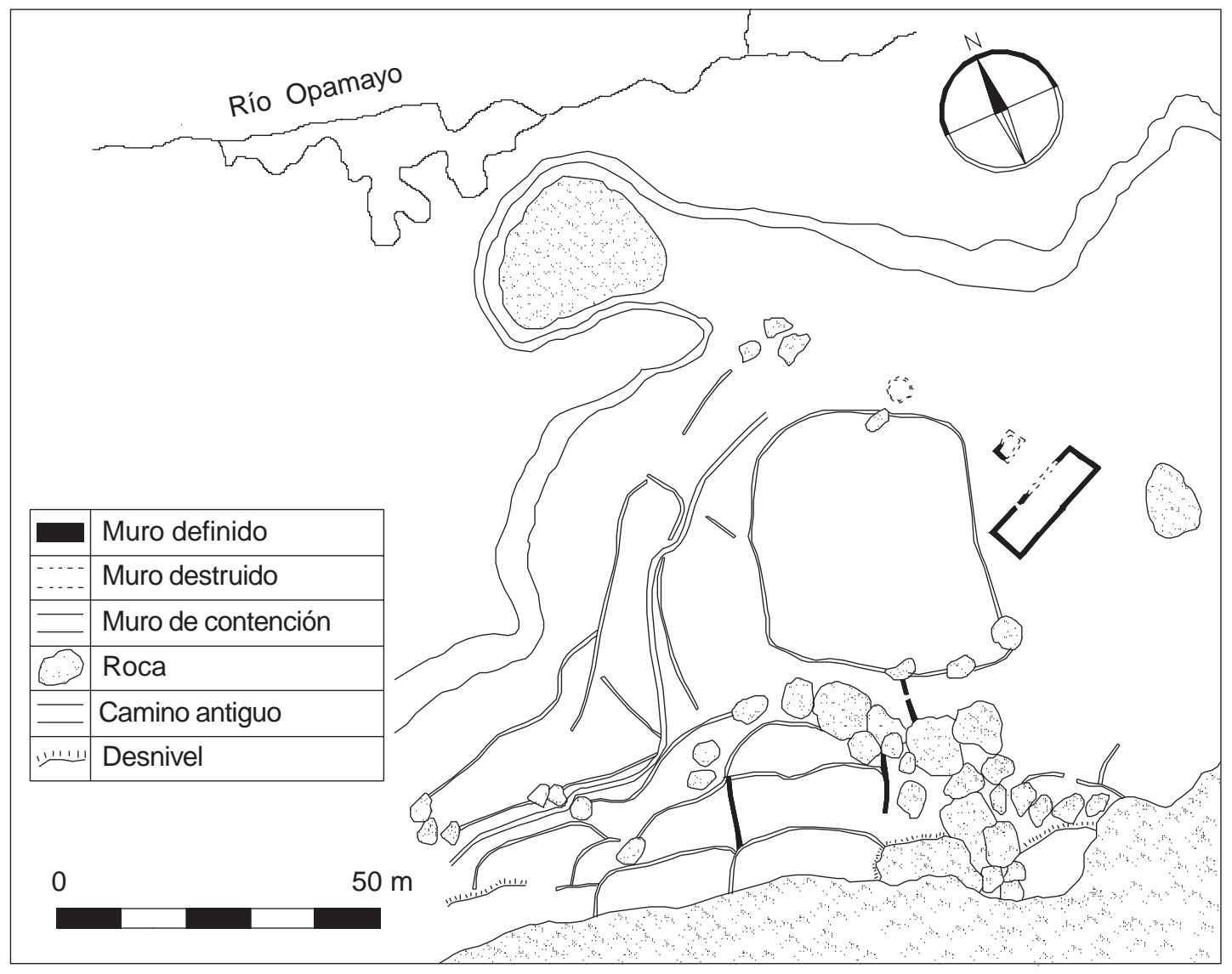

Figura 2. Plano preliminar del sitio de Otorongo (R-32).

Preliminary map of the Otorongo site (R-32). 
conjunto de terrazas artificiales sobre las cuales se observan muros rectos de piedra y barro perpendiculares al eje principal de las terrazas y que dividen los espacios y restringen la circulación entre ellos. Hacia el lado oriental del sitio se aprecian terrazas más amplias en una de las cuales se levantan dos estructuras de planta rectangular y una circular. Esta última mide cuatro metros de diámetro y ha sido reducida actualmente al nivel de sus bases ${ }^{11}$.

La estructura rectangular mayor presenta un acimut de $66^{\circ}$ con su eje mayor orientado hacia la cumbre del Apohuayhuay, siendo sus dimensiones $18.8 \mathrm{~m}$ de largo y $6 \mathrm{~m}$ de ancho. Está levantada con muros de doble hilera de piedras asentadas en barro y que presentan un espesor promedio de 60 $\mathrm{cm}$. En el lado norte del edificio se observan los restos de un vano de acceso de forma trapezoidal de $85 \mathrm{~cm}$ de ancho en su base; sin embargo, debido a las pésimas condiciones de conservación de la sección oriental de este mismo muro se ignora el número de vanos adicionales. La estructura rectangular menor se localiza a escasos $6 \mathrm{~m}$ al NW de la anterior y presenta un acimut de $60^{\circ}$, con un largo de $4.4 \mathrm{~m}$ y un ancho de $2.80 \mathrm{~m}$. Pese a su avanzado estado de destrucción, aún son claras algunas secciones de sus muros, también de piedra y barro, que cuentan con un espesor promedio de $55 \mathrm{~cm}$. Asimismo, debemos señalar que a unos $35 \mathrm{~m}$ al NW de estos edificios se hallan los restos de un viejo camino empedrado, aparentemente prehispánico (Figura 2), denominado por los lugareños “camino de Juan Surichaca". Esta vía cruza el río Opamayo para coronar el abra entre los macizos de Apohuayhuay y Chontahuayhuay y luego descender hacia la ceja de selva.

Pese a nuestros esfuerzos, no se identificó material alfarero claramente diagnóstico asociado al sitio lo cual dificulta su ubicación cronológica. Sin embargo, si tomamos en cuenta las características de la arquitectura y planificación de los edificios descritos ${ }^{12}$, resulta obvio que corresponderían a cánones Inka (Gasparini y Margolies 1977; Hyslop 1990; Kendall 1976).

\section{La Montaña y el Felino: el Control Inka de las Fronteras en el Alto Ricrán}

La configuración del relieve en los Andes y la diversidad de pisos ecológicos y paisajes existentes han conferido peculiar importancia desde un punto de vista económico e ideológico a las fronteras naturales en tiempos prehispánicos. Rasgos naturales que separaban paisajes de contrastes marcados como montañas elevadas y los bordes de la floresta tropical amazónica fueron símbolos esenciales de frontera, en un sentido temporal y espacial (Fioravanti 1986-87:268). Al respecto, la presencia del nevado de Apohuayhuay en la frontera entre dos regiones naturales diferentes, la sierra de Ricrán y la ceja de selva de Monobamba, es destacada, además, por la existencia de un sitio de muy probable filiación Inka como Otorongo (R-32). El topónimo del mismo y su emplazamiento en las "puertas" de la selva son coherentes con el sentido de transición que tuvo el símbolo del felino en los Andes (Zuidema 1989) y con su connotación como intermediario entre el mundo de los hombres y el mundo sobrenatural, entre el tiempo presente representado por la sierra y el tiempo primordial representado por la selva (Fioravanti 1986-87).

Con el Estado Inka se llevó a una mayor escala el uso del felino como símbolo de poder y control del tiempo y del espacio, relacionado a la imagen del Sol y a la persona del Inka ${ }^{13}$. Particularmente el otorongo o jaguar era considerado ancestro y objeto de veneración asociado a las huacas del Antisuyu como Sawasiray y Pitusiray, montañas que separaban al valle del Urubamba de la selva del Cuzco (Guamán Poma 1980 [1615]:268 [270]). Con esta acotación, la particular asociación entre la montaña de Apohuayhuay y el sitio de Otorongo (R-32) sugiere el despliegue de actividades ceremoniales dirigidas por los Inka en torno a Guayoay vilca, huaca de los Tarama, dentro de las cuales el símbolo del jaguar habría jugado un papel importante. Evidencias de ello serían las características arquitectónicas de los edificios del sitio R-32, su orientación hacia el macizo de Apohuayhuay siguiendo posibles alineamientos astronómicos ${ }^{14}$, su asociación a un gran afloramiento rocoso hacia donde mira la estructura mayor, la presencia de plataformas artificiales que definen espacios abiertos amplios y la existencia de un camino empedrado antiguo que vincula al conjunto con el nevado en mención. Todos estos rasgos, en general, presentan una impresionante similitud con aquellos observados en el santuario Inka edificado en la Isla del Sol, lago Titicaca (Dearborn et al. 1998), a lo cual se suma la persistencia del topónimo otorongo en un paraje emplazado en plena cordillera, a más de $4.000 \mathrm{msm}$. 
Considerando la información arqueológica y etnohistórica presentada al inicio, se hace evidente además, que la ubicación de la montaña arriba señalada está ligada con la zona de frontera entre grupos de posible filiación étnica Xauxa y Tarama, hecho que concuerda con la concepción de las huacas y divinidades en los Andes como puntos de mediación o frontera (Fioravanti 1986-87). De este modo el gobierno del Cuzco podría haber ejercido un control más efectivo sobre las poblaciones del alto Ricrán como parte de una política que consideró, como se ha observado en otras partes del Tahuantinsuyu (D'Altroy 1992; Hyslop 1990; Matos 1995; Morris y Thompson 1985; Schreiber 1993), la inserción de segmentos de las mismas dentro de su aparato administrativo como lo sugiere la presencia diferencial de restos de cultura material Inka en varios asentamientos locales, los mismos que incluyen construcciones levantadas en medio de las aldeas locales alterando la configuración original de los edificios preexistentes ${ }^{15}$ y cerámica del tipo Inka Provincial (D'Altroy 1981, 1992; Matos 1999) identificada en mayor cantidad en los sitios asociados a terrazas de cultivo por debajo de los 4.000 $\mathrm{msm}^{16}$.

\section{Comentarios Finales}

La existencia del sitio de Otorongo (R-32) en asociación con la mayor divinidad de los Tarama, marcador de frontera natural y étnica, plantea algunos problemas sobre los criterios para la ubicación de las instalaciones Inka, particularmente con atención a las zonas de transición o fronteras de carácter tanto espacial como temporal, tal como se puede notar en la importancia que dieron los inkas a varios conceptos de gran arraigo en los Andes que denotan simbólicamente divisiones y límites dentro de relaciones de oposición y complementariedad como tinkuy (González Holguín 1989 [1608]:342-343) y yanantin (González Holguín 1989 [1608]:364). Estas concepciones del espacio y tiempo habrían sido llevadas al plano político con la edificación de sitios ceremoniales asociados a montañas sagradas y de otros de carácter administrativo hacia zonas de frontera étnica como estaría ocurriendo con Tarmatambo (Parsons 1998; Parsons et al. 2000:139), la mayor instalación Inka en Tarma (Matos 1997, $2002)^{17}$. De igual modo, tras una reconsideración etnohistórica y arqueológica de los territorios de otras entidades étnicas en la sierra central del Perú, se puede proponer que los centros provinciales de Huanuco Pampa, Pumpu y Hatun Xauxa estarían ocupando zonas de frontera étnica ${ }^{18}$. Así se hace evidente que los inkas, lejos de implementar una política orientada hacia la creación de una homogeneidad política, cultural e ideológica, optaron por una administración de la diversidad ecológica, cultural y étnica de las poblaciones andinas, con la imposición de sus símbolos sobre los elementos sagrados de la geografía local, como montañas o huacas de los grupos conquistados, asegurando de este modo el dominio ideológico sobre ellos (Morris 1998). En el caso del alto Ricrán estas propuestas parecen ser contrastables, pues las evidencias materiales Inka vinculadas a actividades bélicas y militares son prácticamente inexistentes, como se ha señalado también para otras regiones del Tahuantinsuyu (Morris 1998), apuntando por el contrario hacia una coerción ideológica y política como deja entrever Pachacuti Yamqui (1995 [1613]) cuando se refiere a campañas de conquista que incluían la captura de las huacas regionales. Mayores investigaciones en el sitio de Otorongo y en la cuenca del río Ricrán contribuirán a una mejor aproximación a este problema.

Agradecimientos. Nuestras investigaciones en el alto Ricrán fueron financiadas por la Municipalidad Distrital de Ricrán y contaron con la autorización del Instituto Nacional de Cultura (RDN $\mathrm{N}^{\circ}$ 918/INC) y los auspicios del Centro de Estudios "Julio Espejo Núñez" de Jauja y el Instituto del Bien Común de Lima, a los cuales expresamos nuestra gratitud. También agradecemos profundamente a Carmen Arellano, Ramiro Matos, Charles Hastings y José Pino por su apoyo material y sus valiosas observaciones y comentarios sobre el manuscrito original, y a Hugo Marroquín por su ayuda con la preparación del mismo. 


\section{Referencias Citadas}

Albornoz, C.

1967 La instrucción para descubrir todas las guacas del Pirú y sus camayos y haziendas [1582], editado por P. Duviols. Journal de la Société des Américanistes 56:9-39.

Arellano, C.

1988 Apuntes Históricos sobre la Provincia de Tarma en la Sierra Central del Perú. Bonner Amerikanistische Studien 15 , Bonn.

Bonnier, E.

1997 Morfología del espacio aldeano y su expresión cultural en los Andes Centrales. En Archaeologica Peruana 2, editado por E. Bonnier y H. Bischof, pp. 28-41. Sociedad Arqueológica Peruano-Alemana/Reiss-Museum, Mannheim.

Browman, D.

1970 Early Peruvian Peasants: The Culture History of a Central Highlands Valley. Doctoral Dissertation, Department of Anthropology, Harvard University, Cambridge.

Cieza de León, $\mathrm{P}$.

1946 [1533] La crónica general del Perú. En Crónicas de la Conquista del Perú, editado por J. De Riverend, pp. 125497. Editorial Nueva España, México.

Costin, C.

1986 From Chiefdom to Empire State: Ceramic Economy among the Prehispanic Wanka of Highland Peru. Doctoral Dissertation, Department of Anthropology, University of California at Los Angeles, Los Angeles.

1986-87 Cerámica prehispánica tardía del valle de Yanamarca, Junín. Revista del Museo Nacional 48:195-211.

D'Altroy, T.

1981 Empire Growth and Consolidation: the Xauxa Region of Peru under the Incas. Doctoral Dissertation, Department of Anthropology, University of California at Los Angeles, Los Angeles.

1992 Provincial Power in the Inka Empire. Smithsonian Institution Press, Washington and London.

D'Altroy, T. y C. Hastorf

2001 Empire and Domestic Economy. Kluwer Academic/ Plenum Publishers, New York.

Dearborn, D.; M. Seddon y B. Bauer

1998 The sanctuary of Titicaca: where the sun returns to earth. Latin American Antiquity 9:240-258.

DeMarrais, E.

2001 The architecture and organization of Xauxa settlements. En Empire and Domestic Economy, editado por T. D'Altroy y C. Hastorf, pp. 115-153. Kluwer Academia/ Plenum Publishers, New York.

Earle, T., T. D'Altroy, C. Hastorf, C. Scott, C. Costin, G. Russell y E. Sandefur

1987 Archaeological Field Research in the Upper Mantaro Valley, Peru: 1982-1983. Investigations of Inka Expansion and Exchange. Monograph 28, Institute of Archaeology, University of California at Los Angeles, Los Angeles.

Espinoza, W.

1973 Enciclopedia Departamental de Junín 1. Editorial San Fernando, Huancayo.

Estete, M.

1946 [1533] La relación del viaje que hizo el Señor Capitán Hernando Pizarro por mandado del señor gobernador, su hermano, desde el pueblo de Caxamalca a Parcama y de allí a Jauja. En Crónicas de la Conquista del Perú, editado por J. De Riverend, pp. 90-108. Editorial Nueva España, México.

Favre, $\mathrm{H}$.

1983 Introducción. En Asto: Curacazgo Prehispánico de los Andes Centrales, editado por D. Lavallée y M. Julien, pp. 11-23. Instituto de Estudios Peruanos, Lima.

Fioravanti, A.

1986-87 El simbolismo de frontera en los Andes. Revista del Museo Nacional 48:251-286.

Grosboll, S.

1993 ...And he said in the time of the Ynga, they paid tribute and served the Ynga. En Provincial Inca, editado por M. Malpass, pp. 44-76. University of Iowa Press, Iowa City.

Gasparini, G. y L. Margolies

1977 Arquitectura Inka. Centro de Investigaciones Históricas y Estéticas, Facultad de Arquitectura y Urbanismo, Universidad Central de Venezuela, Caracas.

González Holguín, D.

1989 [1608] Vocabulario de la Lengua General de todo el Perú llamada Lengua Quechua o del Inca. Universidad Nacional Mayor de San Marcos, Lima.

Guamán Poma de Ayala, F.

1980 [1615] El Primer Nueva Corónica y Buen Gobierno, editado por J. Murra, R. Adorno y J. Urioste. Editorial Siglo Veintiuno, México.

Hastings, C.

1985 The Eastern Frontier: Settlement and Subsistence in the Andean Margins of Central Peru. Doctoral Dissertation, Department of Anthropology. University of Michigan, Ann Arbor.

1987 Implications of Andean verticality in the evolution of political complexity: a view from the margins. En The Origins and Development of the Andean State, editado por J. Haas, S. Pozorski y T. Pozorski, pp. 145-157. Cambridge University Press, Cambridge.

Hastorf, C., T. Earle, H. Wright, L. LeCount, G. Russell y E. Sandefur

1989 Settlement archaeology in the Jauja Region of Peru. Evidence from the Early Intermediate period through the Late Intermediate period. A Report on the 1986 Field Season. Andean Past 2:81-129.

Hyslop, J.

1990 Inca Settlement Planning. University of Texas Press, Austin.

Kendall, A.

1976 Descripción e Inventario de las Formas Arquitectónicas Inca. Revista del Museo Nacional 42:13-96.

Lavallée, D.

1967 Types ceramiques des Andes Centrales du Perou (Periode Intermediaire récente). Journal de la Sociéte des Americanistes 56:323-364.

LeBlanc, C.

1981 Late Prehispanic Huanca Settlement Patterns in the Yanamarca Valley, Peru. Doctoral Dissertation, Department of Anthropology. University of California at Los Angeles, Los Angeles. 
LeVine, T.

1985 Inka Administration in the Central Highlands: A Comparative Study. Doctoral Dissertation, Department of Anthropology, University of California at Los Angeles, Los Angeles.

Lumbreras, L.

1960 Esquema Arqueológico de la Sierra Central del Perú. Revista del Museo Nacional 28:64-117.

Matos, R.

1959 Los Wanka: datos históricos y arqueológicos. Actas y Trabajos del II Congreso Nacional de Historia del Perú: Época Prehispánica, Tomo 2:187-210. Centro de Estudios Histórico-Militares del Perú, Lima.

1994 Pumpu: Centro Administrativo Inka de la Puna de Junín. Editorial Horizonte, Lima.

1995 Los Inka de la Sierra Central del Perú. Revista de Arqueología Americana 8:159-190.

1997 Incas y etnias regionales en Junín: Una visión arqueológica. En Arqueología, Antropología e Historia en los Andes. Homenaje a María Rostworowski, editado por R. Varón y J. Flores, pp. 397-413. Instituto de Estudios Peruanos, Banco Central de Reserva del Perú, Lima.

1999 La cerámica Inca. En Los Incas. Arte y Símbolos, Colección Arte y Tesoros del Perú, pp. 109-165. Banco de Crédito del Perú, Lima.

2002 El awana wasi de Tarmatambo: una aproximación etnoarqueológica. En El Hombre y los Andes. Homenaje a Franklin Pease G. Y. Tomo II, editado por J. Flores y R. Varón, pp. 679-694. Pontificia Universidad Católica del Perú, Lima.

Matos, R., C. Arellano y D. Brown

1996 Asentamientos inka en Chakamarka y Tarmatambo (departamento de Junín). Problemas y criterios de interpretación para la reconstrucción de una provincia Inka. I Encuentro Internacional de Peruanistas, Tomo I: 181193. Universidad de Lima / Fondo de Cultura Económica, Lima.

Morris, C.

1998 Inka strategies of Incorporation and Governance. En Archaic States, editado por G. Feinman y J. Marcus, pp. 293-309. School of American Research, Santa Fe, New México.

Morris, C. y D. Thompson

1985 Huánuco Pampa: An Inka City and its Hinterland. Thames and Hudson, London.

Pachacuti Yamqui, J.

1995 [1613] Relación de Antigüedades de este Reino del Perú, editado por C. Araníbar. Fondo de Cultura Económica, Lima.

Parsons, J.

1998 A regional perspective on Inka impact in the sierra central, Peru. Tawantinsuyu 5:153-159.

Parsons, J. y C. Hastings

1988 The Late Intermediate period. En Peruvian Prehistory, editado por R. Keatinge, pp. 190-229. Cambridge University Press, Cambridge.
Parsons, J.; C. Hastings y R. Matos

1997 Rebuilding the state in highland Peru: Herder-cultivator interaction during the Late Intermediate period in the Tarama-Chinchaycocha Region. Latin American Antiquity 8 (4):317-341.

2000 Prehispanic Settlement Patterns in the Upper Mantaro and Tarma Drainages, Junin, Peru. Volume I. The Tarama-Chinchaycocha Region. Memoirs of the Museum of Anthropology $\mathrm{N}^{\mathrm{o}} 34$, University of Michigan, Ann Arbor.

Perales, $M$.

2003 Una propuesta para la reconstrucción del mosaico étnico prehispánico tardío en la sierra central del Perú. Manuscrito en posesión del autor.

Perales, M. y A. Rodríguez

2003 Asentamientos prehispánicos tardíos en la puna de Chongos Bajo (Chupaca-Junín). Unay Runa 6:55-59.

Pino, J.

2003 Observatorios y alineamientos astronómicos en el Tampu Inka de Huanuco Pampa, Perú. Ponencia presentada en el Symposium Arq-13 'Etno y Arqueoastronomía en las Américas', $51^{\circ}$ Congreso Internacional de Americanistas, Santiago.

Pizarro, $\mathrm{P}$.

1965 [1571] Relación del Descubrimiento y Conquista de los Reinos del Perú. Crónicas del Perú, Tomo V, editado por Juan Pérez de Tudela Bueso, pp. 159-242. Biblioteca de Autores Españoles 168, Ediciones Atlas, Madrid.

Pulgar, J.

1967 Geografía del Perú: Las Ocho Regiones Naturales del Perú. Universidad Nacional Mayor de San Marcos, Lima.

Schreiber, K.

1993 The Inca occupation of the Province of Andamarca Lucanas. En Provincial Inca, editado por M. Malpass, pp. 77-116. University of Iowa Press, Iowa City.

Tosi, J.

1960 Zonas de vida natural en el Perú. Instituto Interamericano de Ciencias Agrícolas de la OEA, Zona Andina, Proyecto 39. Programa de Cooperación Técnica, Boletín $\mathrm{N}^{\mathrm{o}} 5$, Lima.

Vega, A.

1881 [1582] La descripción que se hizo en la provincia de Xauxa por la instrucción de Su Majestad que á la dicha provincia se invió de molde. Relaciones Geográficas de Indias, Tomo I, pp. 79-95. Ministerio de Fomento del Perú, Madrid.

Xerez, F.

1968 [1533] Verdadera relación de la conquista del Perú y Provincia del Cuzco, llamada Nueva Castilla. Biblioteca Peruana Tomo I, Lima.

Zuidema, T.

1974-76 La Imagen del Sol y la Huaca de Susurpuquio en el Sistema Astronómico de los Incas en el Cuzco. Journal de la Société des Américanistes 63:199-230.

1989 El león en la ciudad. Símbolos reales de transición en el Cusco. En Reyes y Guerreros. Ensayos de Cultura Andina, compilado por M. Burga, pp. 306-383. Fomciencias, Lima. 


\section{Notas}

1 De acuerdo a la clasificación de Tosi (1960) podemos distinguir las siguientes zonas de vida natural: pradera o bosque húmedo montano, piso subalpino, piso alpino y formación nival.

2 Para una discusión más amplia sobre el punto, ver Arellano (1988) y Parsons et al. (2000).

3 Los Xauxa estuvieron concentrados hacia el extremo norte del valle del Mantaro, incluyendo el valle de Yanamarca, y de acuerdo a las primeras descripciones europeas (v.g. Estete 1946 [1533]; Xerez 1968 [1533]) se distinguían de los Huanca mediante el uso de una especia de vincha de color rojo que llevaban en la cabeza.

4 En la literatura arqueológica los Xauxa y Huanca han sido confundidos constantemente, debido a la similitud observada en su cultura material como la construcción de edificios circulares de piedra como viviendas y el uso de géneros similares de cerámica (D’Altroy 1992; Earle et al. 1987). Sin embargo, en los últimos años ha habido reconsideraciones al respecto (ver D'Altroy y Hastorf 2001).

5 Ello se hace particularmente evidente en la edificación de estructuras circulares de piedra como viviendas, la ubicación de sus asentamientos en posiciones defensivas como cumbres elevadas y el uso de la cerámica Mantaro Base Roja.

6 Según Arellano (1988:32), los curacas de Tarma perdieron estas propiedades a raíz de embargos, ventas forzadas, donaciones e invasiones como la que sufrió la Hacienda Yuracmayo a manos de indígenas de Ricrán.

7 Sin embargo, destaca la presencia de áreas de circulación o pequeños corredores entre las distintas estructuras circulares, tal como se aprecia en los sitios de Culimalca (R-5) o Ricramarca (R-10).

8 Según estos autores, los mencionados edificios rectangulares de dos niveles habrían servido como depósitos para almacenar principalmente productos agrícolas (Hastings 1987; Parsons et al. 2000).

$9 \quad \mathrm{Al}$ respecto es importante señalar que en varios sitios del alto Ricrán también se encuentran, aunque en cantidades menores, fragmentos de cerámica que parecen relacionarse con los tipos Wanka Rojo, Alfarería con Temperante de Andesita y Autoengobado Micáceo (Costin 1986, 198687), todos ellos vinculados con los grupos étnicos Xauxa y Huanca del valle del Mantaro.

10 Este es el caso de los sitios de Huaychaomarca (R-35) y Taurishnioc (R-29).
11 En general todas las estructuras han sido afectadas seriamente en tiempos modernos, debido a su reutilización como corrales por parte de los pastores contemporáneos de Ricrán y Yuracmayo. Sin embargo, los muros aún se levantan hasta una altura de $1.2 \mathrm{~m}$.

12 Aquí consideramos principalmente el tipo de planta, la disposición espacial, las dimensiones de los edificios, el espesor de los muros (mucho mayor al promedio de los muros de los edificios de tradición local) y la presencia en la estructura rectangular mayor de vanos de acceso sólo en su lado norte.

13 Al respecto la arquitectura Inka muestra evidencias bastante elocuentes como es el caso de Huanuco Pampa en cuyo sector oriental se exhiben felinos tallados en altorrelieve en una serie de pórticos de fina cantería que siguen un eje equinoccial (Pino 2003).

14 Al menos en el caso del edificio rectangular mayor se observa un alineamiento solsticial claro, con un acimut de $66^{\circ}$ en el eje mayor de la estructura. Referencias de instalaciones destinadas a ritos en fechas del solsticio de junio y vinculadas al símbolo del felino, como el templo de Chuquicancha, han sido previamente señaladas para el caso del Cuzco (ver Zuidema 1974-76).

15 Ello se observa claramente en los sitios de Chanchan (R-7) y Pariamarca (R-17).

16 Sobre el particular, Parsons (1998) ha señalado que en la región de Tarma y Chinchaycocha los asentamientos locales del Horizonte Tardío se localizan a altitudes más bajas que los del período anterior, con el aparente abandono de los sitios emplazados sobre las cumbres de cerros elevados.

17 Aquí debemos destacar que, según LeVine (1985), Tarmatambo carece de elementos arquitectónicos estatales esenciales de carácter ceremonial como el ushnu.

18 Basándonos en una revisión de la literatura disponible, existirían indicios para señalar que Huanuco Pampa se localizó en una frontera entre los Wamali y los Chupachu (Morris y Thompson 1985), en tanto que Pumpu se emplazó en una zona fronteriza entre los Chinchaycocha y los Yaro (Matos 1994; Matos, Arellano y Brown 1996). En el caso de Hatun Xauxa hay indicios para proponer que el sitio fue edificado muy cerca de la tradicional zona de frontera entre los Xauxa y Huanca (Vega 1881 [1582]; Perales 2003). 